\title{
Prediction of fractures in perimenopausal women: a comparison of dual energy $x$ ray absorptiometry and broadband ultrasound attenuation
}

\author{
A Stewart, D J Torgerson, D M Reid
}

\begin{abstract}
Objective-To consider whether bone mineral density (BMD) measurements can predict traumatic fractures occurring in perimenopausal women.

Methods-One thousand perimenopausal women called up for screening underwent both dual energy $x$ ray absorptiometry (DXA) of the spine and hip, and broadband ultrasound attenuation (BUA) of the heel. Two years later, they were sent a questionnaire to discover those who had since had a fracture, and compare them with those who had not.
\end{abstract}

Results-About $2 \%$ of the women had sustained a fracture in the two years since attendance for screening. Fractures in this age group can be predicted weakly, but significantly, by bone mass measurements using DXA and BUA (odds ratios from 1.4 to $2 \cdot 1)$. The lumbar spine appeared to be one of the best predictive sites (odds ratio for 1 SD reduction in BMD $2 \cdot 1$ (95\% confidence interval $1 \cdot 2$ to $3 \cdot 8)$ ), but no significant differences were found between the areas under the curve in receiver operator characteristic (ROC) analysis.

Conclusion-In this preliminary study it appeared that bone mass measurements are predictive of perimenopausal traumatic fractures in addition to postmenopausal fractures related to osteoporosis. DXA of the lumbar spine did not perform significantly better than BUA. The number of fractures occurring was low, however, and further long term follow up is required to confirm the finding.

Osteoporosis
Research Unit,
Aberdeen Royal
Hospitals,
Woolmanhill Hospital,
Aberdeen,
United Kingdom
A Stewart
D M Reid
Health Economics
Research Unit,
University of
Aberdeen,
Aberdeen,
United Kingdom
D J Torgerson
Correspondence to:
Mrs Alison Stewart,
Osteoporosis Research Unit,
Aberdeen Royal Hospitals,
Woolmanhill Hospital,
Aberdeen AB9 1GS,
United Kingdom.
Accepted for publication
6 October 1995

(Ann Rheum Dis 1996; 55: 140-142)

Population screening for osteoporosis risk remains contentious. ${ }^{1}$ However, treatments such as hormone replacement therapy (HRT) are frequently targeted at those in the perimenopause or early postmenopause years, to prevent osteoporotic fractures. Women with non-osteoporotic fractures early in life are at greater risk of sustaining an osteoporotic fracture later in life. ${ }^{2}$ If those women likely to sustain such a fracture could be identified, prophylactic treatment could be offered at an early stage.

Both dual energy $x$ ray absorptiometry (DXA) ${ }^{3}$ and broadband ultrasound attenuation (BUA) ${ }^{4}$ are techniques that have been shown to be predictive of hip fracture, but their relative predictive ability has not yet been examined. In recent case control studies of hip fracture patients, BUA and DXA were equally effective at discriminating prospectively between cases and controls, ${ }^{56}$ and a combination of the two techniques may prove to be significantly better than DXA alone to predict hip fracture risk.

The aims of this study was to compare the predictive abilities of BUA and DXA with respect to perimenopausal fractures.

\section{Patients and methods}

As part of a research programme evaluating the measurement of perimenopausal bone mass as a screening tool for future osteoporosis risk, women were selected at random from a population based register and invited to attend for scanning. ${ }^{7}$ The women reported in this paper were all aged between 45 and 49 years at the time of measurement and lived within a 20 mile radius of Aberdeen. A follow up questionnaire was sent to all patients two years after the measurements were made, and included questions relating to any falls or fractures.

DXA scans of the spine (L2-4), left hip (neck of femur, greater trochanter, and Wards area) were performed to determine bone density at those sites (Norland XR-26).

In 1000 of the women who consented, an additional BUA scan of the os calcis was performed, using a Walker Sonix UBA 575 machine (Walker Sonix, MA, USA). The coefficient of variation of the technique in our hands was $2 \cdot 6 \%$ (range $0-6 \cdot 05 \%$ ) when seven women aged 21 to 51 years were measured, and $3.98 \%$ (range $0-13 \cdot 2 \%$ ) when elderly patients aged between 71 and 92 years (mean 78.7 years) were measured twice in succession with removal of the foot from the scanner between each scan.

Age, standing height, weight (indoor clothing, no shoes) and years postmenopause were recorded. The menopause was defined as the date of the last period, and women were classified as postmenopausal if their last period was six months or more before the date of attendance.

Independent $t$ tests were used to determine the significance of any differences between groups. $x^{2}$ and Fisher's exact test were used to test differences between non-parametric data. Receiver Operator Characteristic (ROC) analysis $^{8}$ was used to identify which technique(s) had the best predictive ability. The SPSS-PC (Chicago, Ill, USA) statistical 
Differences in bone mass measurements between fracture and non-fracture groups

\begin{tabular}{|c|c|c|c|c|}
\hline Variable & $\begin{array}{l}\text { Fracture } \\
\text { group }\end{array}$ & $\begin{array}{l}\text { Non-fracture } \\
\text { group }\end{array}$ & $t$ & $p$ \\
\hline \multicolumn{5}{|l|}{ DXA } \\
\hline L2-4 & $0.97(0.13)$ & $1.07(0.16)$ & $2 \cdot 65$ & 0.008 \\
\hline Neck of femur & $0.86(0.13)$ & $0.89(0.12)$ & $0 \cdot 85$ & 0.395 \\
\hline Trochanter & $0.70(0.10)$ & $0.72(0.11)$ & $0 \cdot 88$ & 0.377 \\
\hline Wards area & $0.78(0.17)$ & $0.85(0.15)$ & 1.94 & 0.053 \\
\hline \multicolumn{5}{|l|}{$\mathrm{Z}$ score } \\
\hline BUA & $-0.332(1.027)$ & $0.000(1.00)$ & $1 \cdot 35$ & 0.177 \\
\hline L2-4 & $-0.654(0.800)$ & $0.01(1.03)$ & $2 \cdot 65$ & 0.008 \\
\hline Neck of femur & $-0.210(1.010)$ & $-0.01(0.96)$ & 0.85 & 0.395 \\
\hline Trochanter & $-0.19(0.95)$ & $0.02(1.01)$ & $0 \cdot 88$ & 0.377 \\
\hline Wards area & $-0.464(1 \cdot 16)$ & $0.02(1.01)$ & 1.94 & 0.053 \\
\hline
\end{tabular}

Values are mean (SD) where relevant.

package was used in calculating the above statistics. Areas under the ROC curve (AUC) ${ }^{9}$ and their statistical differences were calculated using the Rocker program (Quantic Software, 1993). Z scores were calculated from the control group of patients.

\section{Results}

Among the 1000 women who had both a DXA scan and a BUA scan and were sent a questionnaire two years later, 790 replied and 17 of them reported 18 fractures of varying sites. Eight of the fractures were associated with falls, while the others were associated with more traumatic events, such as road traffic accidents. The non-responders to the questionnaire did not differ from the responders with respect to age, height, weight, years postmenopause, or bone mass measurement at baseline.

Fractures of the following sites were reported: wrist, toes, ribs, bones in foot, humerus, and nose. There were no significant differences between the fracture and non-fracture groups for age (fracture $=46.8$ (SD 1.4) years; nonfracture $=47 \cdot 7 \quad(1 \cdot 4)$ years), height (fracture $=1.60 \quad(0.07) \mathrm{m}$; non-fracture $=1.61$ $(0.06) \mathrm{m}$ ), or weight (fracture $=61.6(11.2) \mathrm{kg}$; non-fracture $=65.9(11 \cdot 7) \mathrm{kg})$. The fracture group included seven women $(41 \cdot 2 \%)$ who were premenopausal and 10 who were post-

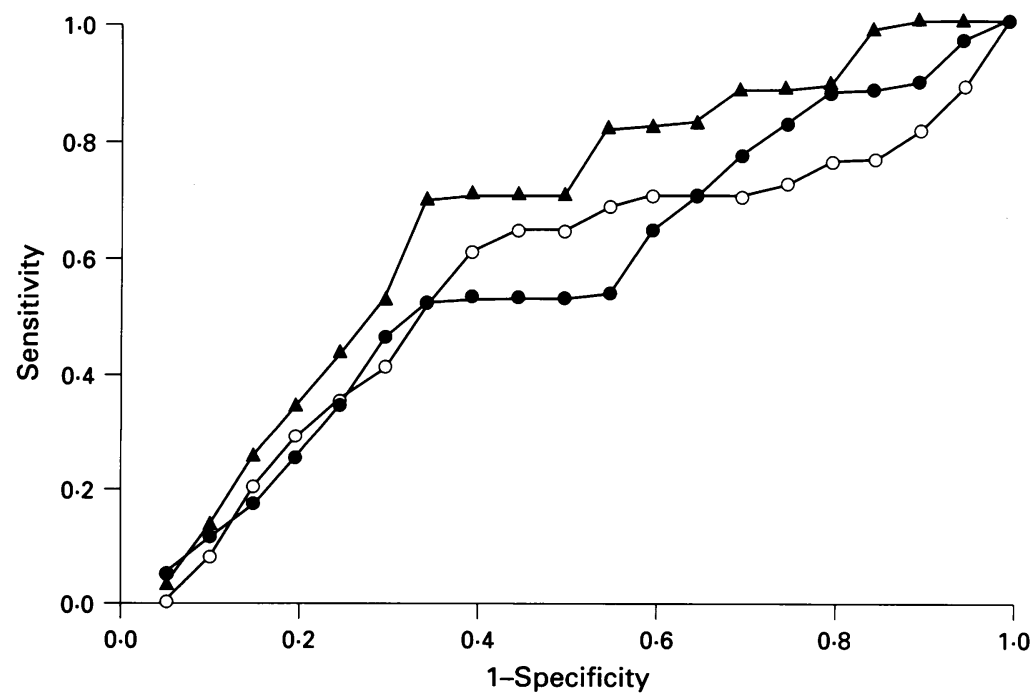

Receiver operator characteristic (ROC) analysis for broadband ultrasound attenuation (O) (area under the curve $(A U C)=0.58$ ) and for dual energy $x$ ray absorptiometry of L2-4 (A) (AUC 0.69), and neck of femur (O) (AUC 0.58). menopausal $(58 \cdot 8 \%$ ), while the numbers of the non-fracture group were $523(67 \cdot 7 \%)$ and 250 women $(32 \cdot 3 \%)$, respectively $\left(\chi^{2}=5 \cdot 28\right.$, $p=0.022)$. There was a trend $(p=0.055)$ towards a difference between fracture and nonfracture groups regarding the number of years postmenopause, the fracture group being a mean 3.6 years since their menopause and the non-fracture group 1.7 years postmenopause, on average.

No significant differences were found for age, height, weight, years postmenopause, or bone mass measurements when comparing those patients whose fracture resulted from a fall from a standing height and those whose fractures resulted from a more traumatic force. Thus all fracture patients were grouped together in the fracture group for further analysis.

All bone mass measurements tended to be lower in the fracture group compared with the non-fracture group, but this reached significance only at the lumbar spine (fracture group $=0.97 \mathrm{~g} / \mathrm{cm}^{2}$; non-fracture group = $1.07 \mathrm{~g} / \mathrm{cm}^{2} ; t=2.65 ; \mathrm{p}=0.008$ ) (table). When the bone mass measurements were adjusted for age, height, weight, and menopausal age, the differences for the spine remained significant $(f=4.465, p=0.035)$, while the significance for the Wards area diminished $(f=1 \cdot 476$, $\mathrm{p}=0.225$ ). Using $\mathrm{z}$ scores instead of raw scores, the findings were similar (table).

Odds ratios were calculated to determine the risk of fracture. For a reduction of $1 \mathrm{SD}$ of bone measurements, the odds ratios (95\% confidence limits) were: BUA $=1.43(1.17$ to $2 \cdot 38)$; L2 $-4=2 \cdot 14(1.22$ to $3 \cdot 76)$, neck of femur $=1.40(1.25$ to 2.36$)$, Wards area $=1.80$ (1.01 to $2 \cdot 85)$.

The area under the curve (AUC) was greatest for DXA L2-4 (AUC $=0.69)$ and least for DXA trochanter (AUC $=0.56$ ), with BUA $(A U C=0.58)$, neck of femur (AUC $=0.58$ ) and Wards area (AUC $=0.64$ ) showing intermediate values (figure). However, none of the differences at any site was significant using the $\mathrm{z}$ statistic. ROC analysis thus showed no one site to be superior in predicting these fractures in this group of perimenopausal women.

\section{Discussion}

Bone densitometers are usually used to measure bone density in order to predict osteoporotic fractures. However, in this study we measured bone mineral density and BUA in perimenopausal women, and followed the women two years later to determine if any fractures had occurred, including traumatic cases. Using the bone measurements it was possible, to a certain degree, to predict even these traumatic fractures in this group of perimenopausal women, with odds ratios up to 2 , which is only slightly less than the odds ratios found in older women when DXA was used to predict hip fracture. ${ }^{3}$ We acknowledge that a major limitation to the study was the small number of fractures occurring in this population to date: only about $2 \%$ of the 
women sustained a fracture during the follow up period of two years, and this is fewer than one would find in an elderly population.

BUA is said to give information regarding the structure of the bone, in addition to its density. ${ }^{1011}$ Unfortunately, the technique was found not to be as good a numerical predictor of perimenopausal fractures as DXA of the spine, but it may provide an independent additional risk factor. The poorer precision values found for ultrasound, compared with DXA, may explain why no significant differences were found between the fracture and non-fracture groups; improving precision for the ultrasound equipment must be a priority.

It has been shown that an axial site such as the spine or hip can predict peripheral fractures, in much the same way as appendicular sites are predictive of spine and hip fractures. ${ }^{12}$ BUA seems to have a predictive ability comparable to that of DXA of the spine and hip. Previous studies ${ }^{56}$ demonstrated that BUA had a discriminatory value similar to that of DXA in patients with hip fractures and those with Colles fractures. ${ }^{13}$ However, in vertebral fracture BUA is not as good a discriminator as DXA of the spine. ${ }^{14}$ BUA may therefore be best applied to determining risk of future hip fracture, while remaining less useful in predicting osteoporotic fractures of the spine.

We conclude that DXA and BUA are not only useful in predicting osteoporotic fractures in the elderly, but also have some use in predicting all fractures, including those caused by traumatic forces in a perimenopausal group of women. One study has shown that those people who sustain non-osteoporotic fractures in early adulthood will continue to sustain fragility fractures later in life: those with a nonosteoporotic fracture (tibial shaft or ankle fracture) before the age of 50 years showed an odds ratio of 1.9 for a future fragility fracture. ${ }^{2}$ Therefore, if it is possible to detect those who have an early non-osteoporotic fracture as a result of even moderate trauma, it may be possible to offer prophylactic treatment and prevent fragility fractures later in life. Early postmenopausal women suffering a traumatic fracture could, perhaps, be considered for bone density assessment to determine their suitability for preventive treatments. The present study was preliminary in nature, and follow up of a larger target population is required.

The authors are grateful for grant support from the Scottish Office Home and Health Department and the Wolfson Foundation. DMR is grateful for continued support from the Arthritis and Rheumatism Council. We thank Ruth Thomas for help with the questionnaire data, and Dr Peter Tothill and Dr help with the questionnaire data, and Dr Peter Tothill and Dr Alison Aven
machine.

1 Effective Health Care. Screening for osteoporosis to prevent fractures. Effective Health Care 1992; No 1.

2 Karlsson M K, Hasserius R, Obrant K J. Individuals who sustain non-osteoporotic fractures continue to also sustain fragility fractures. Calcif Tissue Int 1993; 53: 229-31.

3 Cummings S R, Black D M, Nevitt M C, et al. Bone density at various sites for prediction of hip fractures. Lancet 1993; 341: 72-5.

4 Porter R W, Miller C, Grainger S. Prediction of hip fractures in elderly women: a prospective study. $B M F 1990 ; 301$ : 638-41.

5 Stewart A, Reid D M, Porter R W. Broadband ultrasound attenuation and dual energy x-ray absorptiometry in patients with hip fractures: which technique discriminates fracture risk. Calcif Tissue Int 1994; 54: 466-9.

6 Turner C H, Peacock M, Timmerman L, Neal J M, Johnston C C. Calcaneal ultrasonic measurements discriminate hip fracture independently of bone mass. Osteoporosis Int 1995; 5: 130-5.

7 Garton M J, Torgerson D J, Donaldson C, Russell I T, Reid D M. Recruitment methods for screening programmes: trial of a new method within a regional osteoporosis study. BMF 1992; 305: 82-5.

8 Metz C. Basic principles of ROC analysis. $f$ Nucl Med 1978; 3: 283-8.

9 Hanley J A, McNeil B J. The meaning and use of area under a Receiver Operating Characteristic (ROC) curve. Radiology 1982; 143: 29-36.

10 Langton C M, Evans G P, Hodgskinson R, Riggs C M. Ultrasonic, elastic and structural properties of cancellous
bone. In: Ring E F G, ed. Current research in osteoporosis bone. In: Ring E F G, ed. Current research in osteoporosis and bone mineral measurem

11 Gluer C C, Faulkner K G, Engelke K, Black D, Genant H K. Broadband ultrasound attenuation of the heel and single photon absorptiometry of the heel: do these measure different entities? F Bone Miner Res 1991; 6: S169.

2 Wasnich R D, Ross P D, Heilbrun L K, Vogel J M. Prediction of postmenopausal fracture risk with use of bone mineral measurements. Am f Obstet Gynecol 1985; 153: 745-51.

13 Douglas AS, Robins S P, Stewart A, Reid D M, Hutchison JD, Porter R W. Carboxylation of osteocalcin in postmenopausal osteoporotic women following Vitamin $\mathrm{K}$ menopausal osteoporotic women following

14 Stewart A, Felsenberg D, Kalidis L, Reid D M. Vertebral fractures in men and women: How discriminative are bone mass measurements? Br $\mathscr{F}$ Radiol 1995; 68: 614-20. 